\title{
Reported Stigma and Discrimination by People with a Diagnosis of Schizophrenia - CORRIGENDUM
}

\author{
D. Rose, R. Willis, E. Brohan, N. Sartorius, C. Villares, K. Wahlbeck, G. Thornicroft and for the \\ INDIGO Study Group
}

doi:10.1017/S2045796011000254, Published by Cambridge University Press, 21 March 2011.

The acknowledgements section was not included in the above publication (Rose et al., 2011). The acknowledgements are provided below.

The authors apologise for this omission.

\section{Acknowledgements}

GT is supported in relation to a National Institute for Health Research (NIHR) Applied Programme grant awarded to the South London and Maudsley NHS Foundation Trust. GT and DR are supported in relation to the NIHR Specialist Mental Health Biomedical Research Centre at the Institute of Psychiatry, King's College London and the South London and Maudsley NHS Foundation Trust.

\section{Reference}

Rose D, Willis R, Brohan E, Sartorius N, Villares C, Wahlbeck K, Thornicroft G (2011). Reported stigma and discrimination by people with a diagnosis of schizophrenia. Epidemiology and Psychiatric Sciences 20, 193-204 doi:10.1017/S2045796011000254. 